\title{
Desenvolvimento e Implementação do Controle Automático de Velocidade Linear e Angular de um Robô Auto Equilibrado Usando Filtro de Kalman e Encoders de Baixa Resolução
}

\author{
Joabe R. Silva*, Ravena S. Monteiro*, Ronaldo A. Oliveira*, Daniel Fernando T. Gamarra**, Marco Antonio S. L. Cuadros* \\ * Instituto Federal do Espírito Santo - Campus Serra \\ Rodovia ES 010, Km 6,5, 29173-087 Manguinhos, Serra, ES, Brasil \\ E-mails: joaberuella@gmail.com,ravenaa.soares@hotmail.com,ronaldo.oliveira@ifes.edu.br, marcoantonio@ifes.edu.br \\ ** Universidade Federal de Santa Maria - Coordenadoria de Engenharia de Controle e Automação \\ Av. Roraima, 1000 - Camobi, 97105-900, Santa Maria, RS, Brasil \\ E-mail: daniel.gamarra@ufsm.br
}

\begin{abstract}
In this paper the construction and structure for the implementation of linear and angular velocity controllers of a selfbalanced differential robot using the NI myRIO embedded system and the LabVIEW software are presented. Each of the two wheels of the robot has a PID speed controller, and a low-resolution encoder, so this makes the speed measurement have abrupt variations, consequently compromising the quality of the action of the controllers in the process. To solve such problem, we used the one-dimensional Kalman filter. In addition to the implementation of the linear and angular velocity controllers and the wheel speed controllers of the robot, the control system must act in self-balancing. At the end, practical results, conclusions and recommendations are presented.
\end{abstract}

Resumo: Neste artigo são apresentadas a construção e uma estrutura para a implementação de controladores de velocidade linear e angular de um robô diferencial auto equilibrado utilizando o sistema embarcado NI myRIO e o software LabVIEW. Cada uma das duas rodas do robô possui um controlador de velocidade PID, e um encoder de baixa resolução, portanto, isto faz que a medição da velocidade tenha variações bruscas, consequentemente, comprometendo a qualidade da ação dos controladores no processo. Para resolver tal problema, utilizou-se o Filtro de Kalman de uma dimensão. Além da implementação dos controladores de velocidade linear, angular e das velocidades das rodas; o sistema de controle deve também atuar nas rodas para manter o equilíbrio. Ao final, são apresentados resultados práticos, conclusões e recomendações.

Keywords: System Control, Mobile Robotics, Self-Balancing Robot, Kalman Filter.

Palavras-chaves: Controle de Sistemas, Robótica móvel, Robô Auto Equilibrado, Filtro de Kalman.

\section{INTRODUÇÃO}

Devido à existência de trabalhos perigosos e que põem em risco a integridade física de pessoas e equipamentos e à necessidade de aumentar a produção industrial, desenvolveuse nos últimos anos uma variedade de robôs para diferentes aplicações. Dentre as áreas que recebem investimentos para a utilização de robôs, estão: automação de processos da produção industrial, realização de trabalhos em áreas insalubres, substituição de pessoas nos trabalhos que envolvem esforços repetitivos, vigilância e segurança territorial, entrega de produtos e logística, entre outros (Rosário 2005).

Os robôs são basicamente subdivididos em dois tipos: robôs estacionários e robôs móveis. Como o próprio nome diz, os robôs estacionários são aplicados em situações onde não há a necessidade de locomoção do robô no ambiente de trabalho, como exemplo, tem-se o robô manipulador, que é comumente aplicado nas linhas de montagem de veículos e lembra um braço humano montado em uma base. Os robôs móveis são categorizados de acordo com o ambiente em que ele irá se locomover, sendo: robôs terrestres (com rodas ou pernas), robôs aquáticos, robôs aéreos e os robôs híbridos. $\mathrm{Na}$ subcategoria robôs com rodas, eles podem ter de uma até várias rodas (Dobra 2015).

O robô de duas rodas, que é usado neste trabalho, é caracterizado por ser desafiador para a engenharia de controle, pois deriva do problema de equilibrar o pêndulo invertido e vem sendo estudado há pelo menos duas décadas (Grasser et al. 2002; Ha \& Yuta 1994; Sadeghian \& Masoule 2016;
Shiroma et al. 1996). Ele possui uma vasta aplicação no ensino e pesquisa, possibilitando o estudo de diversos tipos de controladores, pois se trata de um sistema não-linear e instável. Uma aplicação comercial bastante conhecida do robô de duas rodas é no transporte de pessoas, e um deles é o Segway PT (Segway 2018). Outras aplicações que possuem o princípio de funcionamento semelhante ao robô de duas rodas é a estabilização de foguetes na fase de lançamento (ou pouso) e no controle de posição de satélites.

Garantir que um robô saia de uma posição e chegue a outra de forma automática é conhecido como navegação autônoma. Em um robô de duas rodas, a navegação pode ser feita mediante a implementação de controladores de velocidade de deslocamento, de orientação e de equilíbrio, todos atuando concomitantemente no robô. Por ser um trabalho desafiador, muitos algoritmos de controle já foram estudados e aplicados em robôs de duas rodas e, dentre eles, o controlador PID (Proporcional-Integral-Derivativo) continua sendo uma alternativa amplamente utilizada (Sadeghian \& Masoule 2016), (Prasetio 2016), (Kumar, Vijaydeep \& Gupta 2017) e (Sarathy et al. 2018).

A estabilidade do robô de duas rodas se dá quando o mesmo fica paralelo ao eixo vertical. Os desafios aumentam quando também se deseja controlar o deslocamento do robô, ou seja, ele deverá mover-se para um determinado ponto ou determinada direção, mantendo o seu equilíbrio.

Sendo assim, este trabalho tem por objetivos realizar a construção de um robô de duas rodas com encoders de baixa resolução, apresentar a modelagem matemática do sistema, implementar controladores que atuem no sistema conseguindo 
manter o robô na posição vertical, e atuar na regulação da velocidade de deslocamento linear e angular.

Este artigo está dividido em 5 seções; na primeira seção apresenta-se a introdução; na segunda seção descreve-se o hardware utilizado; a seção 3 trata do Filtro de Kalman; a quarta seção descreve a estrutura de controle e na quinta seção apresenta-se os testes práticos. Finalmente são discutidas as conclusões e recomendações.

\section{DESCRIÇÃO DO ROBÔ AUTO EQUILIBRADO}
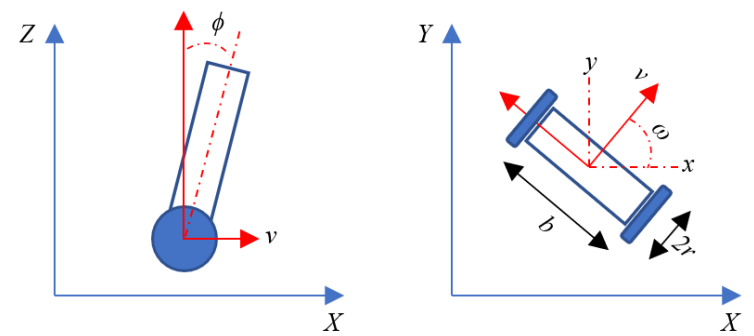

Figura 1 - Representação da estrutura do robô.

O objetivo de controle é regular a velocidade de deslocamento $v$, a de orientação $\omega$ e a inclinação $\phi$ do robô para os valores desejados (Figura 1), $v_{d}, \omega_{d}$ e zero, respectivamente.

A descrição matemática do robô, partindo do pressuposto de que não há deslizamento entre as rodas e o solo, foi feita mediante as forças de equilíbrio e os momentos que atuam sobre ele, onde: $F_{r} F_{l}, H_{r} H_{l}, \tau_{r} \tau_{l}, d_{r} d_{l}, \theta_{r} \theta_{l}, x_{r} x_{l}$ e $d$ são, respectivamente, as forças de interação na plataforma, as forças de atrito, os torques, as forças externas, os ângulos de rotação, os deslocamentos e a distância das rodas direita e esquerda; $\theta$ é o ângulo de orientação; $M_{w}, m$ e $M$ são as massas da roda, do pendulo e da plataforma, respectivamente; $I_{w}, I_{M}$ e $I_{P}$ são, respectivamente, os momentos de inércia da roda em relação ao eixo y, da plataforma sobre o eixo y e da plataforma e do pêndulo em torno do eixo $z ; r$ é o raio da roda; $g$ é aceleração da gravidade; $l$ é a distância do eixo y ao centro de gravidade do pêndulo; $F_{P}$ momento de interação entre o pêndulo e a plataforma em torno do eixo y; $v$ é a velocidade de deslocamento do robô. Da roda esquerda, resulta nas equações de movimento (1) e (2). E (3) e (4) para a da direita.

$$
\begin{gathered}
I_{w} \ddot{\theta}_{l}=F_{l}-H_{l} r \\
M_{w} \ddot{x}_{l}=d_{l}-F_{l}+H_{l} \\
I_{w} \ddot{\theta}_{r}=F_{r}-H_{r} r \\
M_{w} \ddot{x}_{r}=d_{r}-F_{r}+H_{r}
\end{gathered}
$$

As forças de equilíbrio que atuam no pêndulo na direção do eixo $x$ e os momentos em torno do ponto central de gravidade $O$ resultam nas equações (5) e (6),

$$
\begin{aligned}
& -m l \cos (\phi) \ddot{\phi}+m l \dot{\phi}^{2} \sin (\phi)-m \ddot{x}=F_{p} \\
& m l^{2} \ddot{\phi}+m l \cos (\phi) \ddot{x}-m g l \sin (\phi)=M_{p}
\end{aligned}
$$

onde $m l^{2}$ é o momento de inércia do pêndulo em relação ao eixo y. As forças de equilíbrio que atuam na plataforma ao longo da direção do eixo $x$ e momentos sobre o eixo $z$ resultam nas equações $(7)$ e (8).

$$
\begin{gathered}
M \ddot{x}=F_{l}+F_{r}+F_{p} \\
I_{M} \ddot{\phi}=-M_{p}
\end{gathered}
$$

Equilibrar os momentos que atuam na plataforma e no pêndulo sobre o eixo $\mathrm{z}$ resulta na equação (9).

$$
I_{M} \dot{\omega}=b\left(F_{l}-F_{r}\right)
$$

As operações algébricas envolvendo as equações de (1) a (9) resulta nas equações de movimento do robô móvel de duas rodas com um pêndulo invertido, como mostrado nas equações (10), (11) e (12),

$$
\begin{gathered}
\dot{\omega}=\frac{b}{2 r I_{\theta}}\left(\tau_{l}-\tau_{r}\right)+\frac{b}{2 I_{\theta}}\left(d_{l}-d_{r}\right) \\
\ddot{x}=m l \sin (\phi) \frac{I_{\phi} \dot{\phi}^{2}-m l g \cos (\phi)}{\Omega_{0}}+ \\
\frac{I_{\phi}\left(\tau_{l}+\tau_{r}\right)}{\Omega_{0} r}+\frac{I_{\phi}\left(d_{l}-d_{r}\right)}{\Omega_{0}} \\
\ddot{\phi}=m l \sin (\phi) \frac{M_{x} g-m l \cos (\phi) \dot{\phi}^{2}}{\Omega_{0}}- \\
m l \cos (\phi)\left[\frac{\left(\tau_{l}+\tau_{r}\right)}{\Omega_{0} r}+\frac{\left(d_{l}+d_{r}\right)}{\Omega_{0}}\right] \\
I_{\theta}=I_{p}+\frac{b^{2}}{2}\left(\frac{I_{w}}{r^{2}}+M_{w}\right) ; I_{\phi}=m l^{2}+I_{M} \\
M_{x}=M+m+2\left(\frac{I_{w}}{r^{2}}+M_{w}\right) \\
\Omega_{0}=M_{x} I_{\phi}-(m l \cos (\phi))^{2}
\end{gathered}
$$

Linearizando as equações de movimento em torno do ponto de operação $(\phi=0)$ e considerando as forças externas iguais a zero $\left(d_{l}=d_{r}=0\right)$, definimos o vetor de variáveis de estados como $\left[\begin{array}{llllll}\phi & \dot{\phi} & x & v & \theta & \omega\end{array}\right]^{T}$. Por fim, o modelo em espaço de estados do sistema é mostrado na equação (16),

$$
\left[\begin{array}{c}
\dot{\phi} \\
\ddot{\phi} \\
v \\
\dot{v} \\
\omega \\
\dot{\omega}
\end{array}\right]=\left[\begin{array}{cccccc}
0 & 1 & 0 & 0 & 0 & 0 \\
t_{1} & 0 & 0 & 0 & 0 & 0 \\
0 & 0 & 0 & 1 & 0 & 0 \\
t_{2} & 0 & 0 & 0 & 0 & 0 \\
0 & 0 & 0 & 0 & 0 & 1 \\
0 & 0 & 0 & 0 & 0 & 0
\end{array}\right]\left[\begin{array}{c}
\phi \\
\dot{\phi} \\
x \\
v \\
\theta \\
\omega
\end{array}\right]+\left[\begin{array}{cc}
0 & 0 \\
t_{3} & 0 \\
0 & 0 \\
t_{4} & 0 \\
0 & 0 \\
0 & t_{5}
\end{array}\right]\left[\begin{array}{l}
\tau_{\phi} \\
\tau_{\theta}
\end{array}\right]
$$

onde

$$
\begin{gathered}
t_{1}=\frac{M_{x} m g l}{\Omega_{0}} ; t_{2}=\frac{-m^{2} l^{2} g}{\Omega_{0}} ; t_{3}=\frac{-m l}{\Omega_{0} r} ; t_{4}=\frac{I_{\phi}}{\Omega_{0} r} ; \\
t_{5}=\frac{b}{2 r I_{\theta}} ; \tau_{\phi}=\left(\tau_{l}+\tau_{r}\right) ; \tau_{\theta}=\left(\tau_{l}-\tau_{r}\right)
\end{gathered}
$$

O hardware do robô utilizado neste trabalho consiste em um sistema embarcado NI myRIO da National Instruments, contendo três conectores com várias entradas e saídas digitais e analógicas, um processador em tempo real ARM Dual Core e um FPGA Xillinx. Outra característica do NI myRIO é poder realizar a troca de dados entre o computador e o robô via WiFi (National Instruments 2018).

Para obter a medição do ângulo de equilíbrio do robô (em relação ao eixo vertical) foi utilizado o sensor de orientação absoluta BNO055, embarcado em uma IMU (Inertial Measurement Unit) da fabricante Adafruit. A comunicação entre o dispositivo e o NI myRIO é realizada por meio do protocolo I2C (Inter-Integrated Circuit).

Os dois motores de corrente contínua, modelo IG-22, são acionados por um drive de potência por meio de sinais PWM (Pulse-Width Modulation) enviados do NI myRIO, assim, é possível variar as velocidades dos dois motores. O driver utilizado é da marca Pololu, modelo MD03A, pois ela é compacta e permite conectar 2 motores diferenciais. Em cada motor é acoplado um encoder rotacional magnético de efeito Hall de dois canais, categorizado como encoder incremental, com resolução de 230ppr (pulsos por revolução).

\section{APLICAÇÃO DO FILTRO DE KALMAN (FK)}

O FK é um algoritmo matemático que produz uma solução 
para o problema de filtragem linear de sinais discretos. $\mathrm{O}$ mesmo vem desempenhando um papel importante em diversas áreas de pesquisas, pois ele possibilita a redução de ruídos em sinais de processos (Bishop \& Welch 2001). O algoritmo permite, via implementações computacionais, estimar os estados $\hat{x}$ de um processo discretizado qualquer, baseado na sua modelagem e na medição $z$.

O algoritmo de Kalman pode ser subdividido em duas etapas: predição e atualização. A etapa de predição é composta pelas equações (18) e (19), a etapa de atualização é composta pelas equações (20), (21) e (22),

$$
\begin{gathered}
\text { Predição: } \\
\hat{x} \leftarrow(A \hat{x}+B u) \\
P \leftarrow\left(A P A^{T}+Q\right) \\
\text { Atualização: } \\
K=P H^{T}\left(H P H^{T}+R\right)^{-1} \\
\hat{x} \leftarrow[\hat{x}+K(z-H \hat{x})] \\
P \leftarrow[(1-K H) P]
\end{gathered}
$$

onde: $A$ e $B$ são matrizes que derivam da modelagem do processo em espaço de estados sendo que, a matriz $A$ relaciona o estado no instante de tempo anterior com o estado atual e a matriz $B$ relaciona a entrada de controle $u$ com o estado $\hat{x} ; P$ é a covariância do erro estimado e $Q$ é a de covariância do ruído do processo; $K$ é o ganho de Kalman; $H$ é a matriz de transformação da medição; $R$ é o erro da medição; cada seta $(\leftarrow)$ indica atualização da respectiva variável.

\subsection{O Filtro de Kalman de uma dimensão}

Uma desvantagem do FK é a necessidade de computar inversões de matrizes para estimar vetores de estados multidimensionais, podendo comprometer a implementação desse tipo de filtro em microcontroladores com baixa capacidade de processamento. Uma opção para reduzir a demanda computacional ao usar um FK é reduzir o modelo do sistema para apenas uma variável, portanto, as matrizes são reduzidas a números reais e as inversões de matrizes tornamse operações de divisão. Usando esta abordagem, o algoritmo de Kalman pode ser reescrito seguindo as equações (23), (24), (25), (26) e (27).

$$
\begin{aligned}
& \text { Predição: } \\
& \hat{x} \leftarrow(a \hat{x}+b u) \\
& p \leftarrow(a p a+q) \\
& \text { Atualização: } \\
& K=p h /(h p h+r) \\
& \hat{x} \leftarrow[\hat{x}+K(z-h \hat{x})] \\
& p \leftarrow[(1-K h) p]
\end{aligned}
$$

Daí, tem-se o ODKF ou SDKF (One Dimensional Kalman Filter ou Single Dimensional Kalman Filter), FK de uma única dimensão (Shaowei \& Shanming 2012).

\subsection{O FK aplicado nas medições de velocidades}

Como os encoders rotativos utilizados neste trabalho são de baixa resolução (230ppr), isso gera medições de velocidades linear e angular ruidosas. Como explicado em (Shaowei \& Shanming 2012), isso ocorre devido à baixa resolução dos encoders associado ao baixo tempo de amostragem.
Pela teoria de controle clássico, implementar controladores com ação derivativa, onde a realimentação fornece sinais ruidosos, não é tarefa fácil. Nestas condições, o primeiro pensamento para resolver o problema do ruído é implementar o filtro passa-baixa da classe IIR (Infinite Impulse Response), porém, o mesmo pode inserir atrasos no comportamento do sistema (Shaowei \& Shanming 2012). Outra alternativa é o FK de uma dimensão (SDKF), uma solução factível e de baixo custo computacional que já se mostrou bastante eficaz em (Shaowei \& Shanming 2012) e (del Pino et al. 2018), logo, decidiu-se implementá-lo neste trabalho para melhorar a medição de velocidade de cada motor do robô.

A implementação do SDKF no LabVIEW foi feito seguindo as equações (23), (24), (25), (26) e (27), e mostrada na Figura 2.

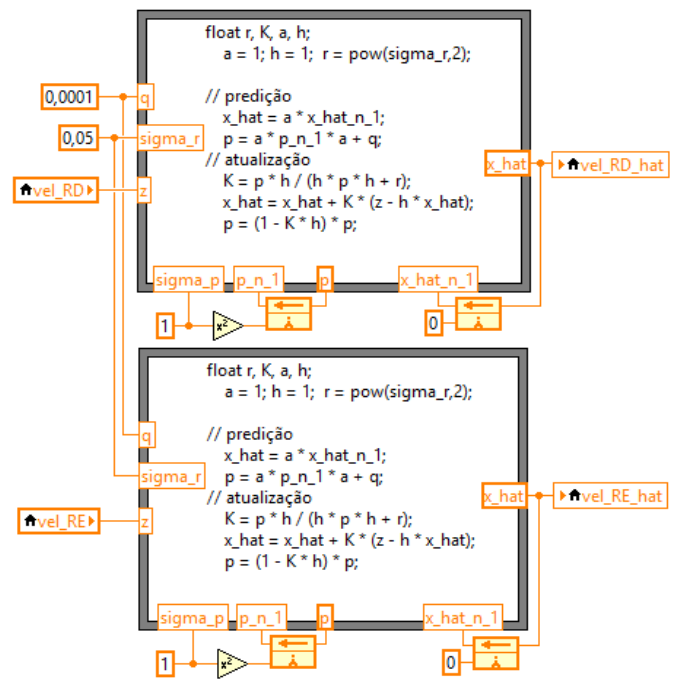

Figura 2 - Implementação no LabVIEW do SDKF.

Nesta implementação, fez-se as seguintes considerações:

- Os erros de medição são aleatórios, logo, pode-se descrevê-los por variância $\left(\sigma^{2}\right)$. A variância dos erros de medição é, na verdade, a incerteza de medição ou erro de medição, portanto, a incerteza de medição $(r)$ é a variância da medição $\left(\sigma_{z}^{2}\right)$. Ela pode ser fornecida pelo fabricante do sensor ou pode ser obtida por procedimento de calibração. Neste trabalho ela será definida por um valor aproximado.

- Como a constante de tempo dos motores elétricos é pequena em comparação com as constantes de tempo do robô, a dinâmica do motor foi considerada constante no modelo. Como resultado, o coeficiente do sistema foi definido como $a=1$. Analogamente, $h=1$. Como foi considerado que o modelo possui dinâmica constante, a estimativa prevista é igual à estimativa atual $(\hat{x} \leftarrow a \hat{x})$.

- A diferença entre a estimação e o valor verdadeiro é o erro da estimação. A tendência é que o erro estimado se torne cada vez menor à medida que mais medições são feitas, convergindo para zero, ou seja, o valor estimado converge para o valor real. Inicialmente, não se sabe qual é o erro de estimação, mas pode-se estimar a incerteza da estimação (sigma_p), representada por $p$.

- A incerteza do modelo dinâmico é chamada de ruído de processo. O ruído do processo produz erros de estimação. A variância de ruído de processo é indicada pela letra $q$, 
logo, para dinâmica constante: $p \leftarrow(a p a+q)$.

Todas estas suposições fazem acreditar que se tem um modelo impreciso, portanto, por tentativa e erro, definiu-se: a variância do ruído do processo como $q=0,0001$; o erro de medição (desvio padrão) é sigma_z $=0,05 \mathrm{~m} / \mathrm{s}$; a incerteza da estimação inicial é sigma_ $p=1 \mathrm{~m} / \mathrm{s}$. Todos estes valores foram superestimados, sendo um indicativo de não confiabilidade no primeiro palpite, pois se os mesmos forem inicializados com valores mais precisos, obtém-se uma convergência mais rápida do FK.

Com o objetivo de testar esses algoritmos, foi dado partida em ambos motores com o duty cycle do sinal PWM em 50\% (metade da tensão média suportada pelos motores), mantendo o sentido de giro e período de amostragem de $5 \mathrm{~ms}$, sumulando um movimento em linha reta. Em termos técnicos, pode-se dizer que foi realizado um teste de resposta ao degrau de velocidade linear em malha aberta. Neste teste, os sinais capturados pelas medições da velocidade de deslocamento da roda direita e esquerda, podem serem vistos na Figura 3.
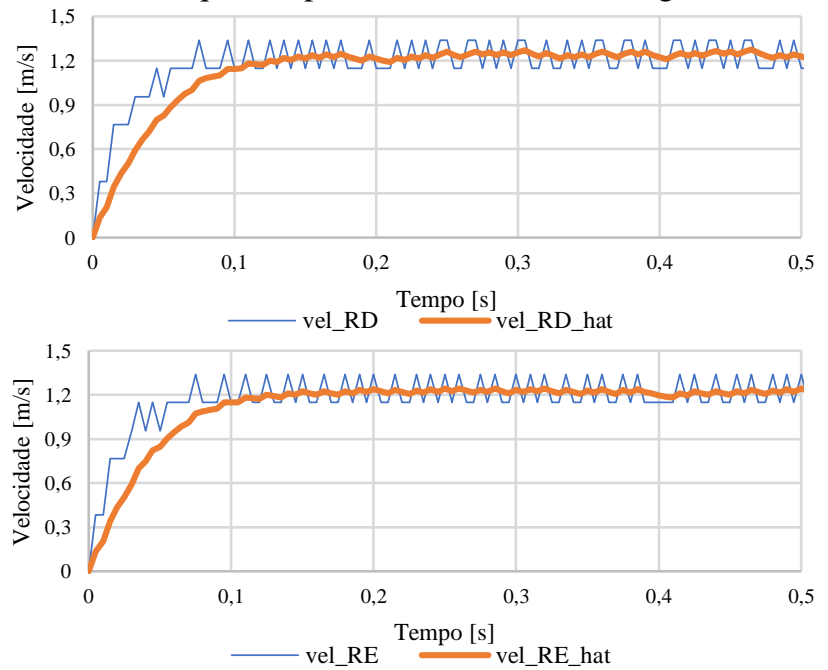

Figura 3 -Resposta ao degrau em malha aberta da velocidade da roda direita (vel_RD) e roda esquerda (vel_RE). As linhas finas são os sinais capturados pelos encoders, as linhas grossas são os mesmos sinais filtrados pelo Kalman (vel_RD_hat e vel_RE_hat).

Os sinais de velocidades apresentados na Figura 3 podem ser utilizados para calcular as velocidades linear e angular do robô em malha aberta, que são obtidas por meio das equações (28) e (29),

$$
\begin{aligned}
v[k] & =\frac{v_{R D}[k]+v_{R E}[k]}{2} \\
\omega[k] & =\frac{v_{R D}[k]-v_{R E}[k]}{b}
\end{aligned}
$$

onde: $v[k]$ e $\omega[k]$ são, respectivamente, a velocidade linear e angular do robô no instante de tempo discreto $k ; b$ é a distância entre as rodas do robô. No LabVIEW, estas equações foram implementadas como se mostra na Figura 4.

Os dados filtrados pelo FK são usados nos cálculos das velocidades linear e angular, um teste é mostrado na Figura 5. Observa-se que o FK proporciona uma melhora significativa para a medição das velocidades linear e angular no robô. Concluído o processamento do sinal de medição, passa-se à próxima etapa, definir os controladores.

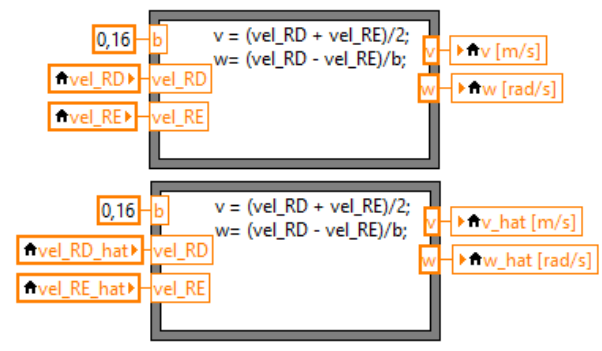

Figura 4-Algoritmo para calcular a velocidade linear e angular.
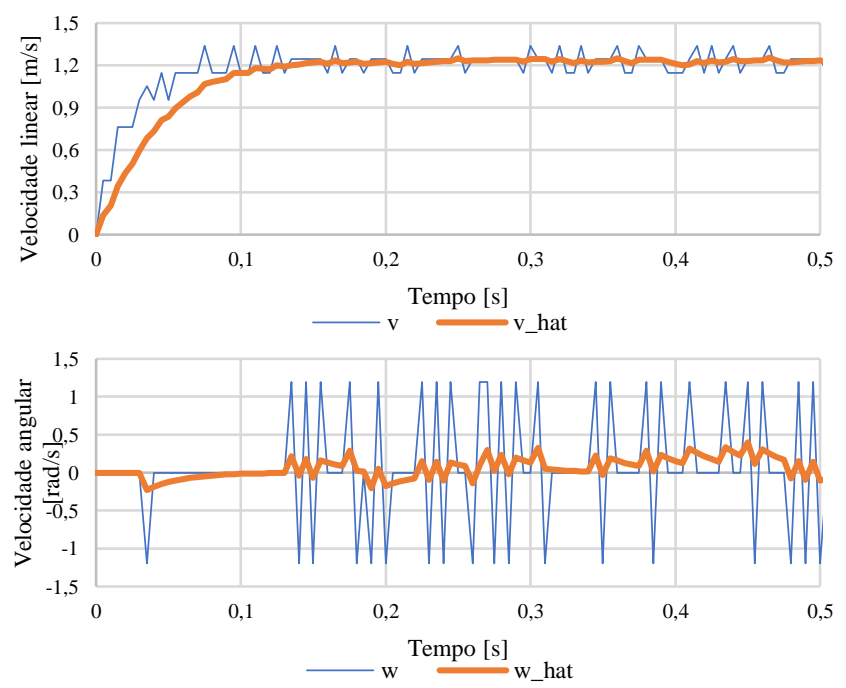

Figura 5 - Resposta ao degrau em malha aberta da velocidade linear (v) e a velocidade angular (w). As linhas finas representam o sinal capturado pelos encoders, as linhas grossas são os respectivos sinais filtrados pelo Kalman (v_hate $w_{-}$hat, respectivamente).

\section{ESTRUTURA DOS CONTROLADORES}

O controlador PID (proporcional, integral e derivativo) é amplamente utilizado em sistemas de controle por realimentação desde a década de 1920 (Campos \& Teixeira 2006). Na equação (30) é apresentada a estrutura padrão ISA deste controlador (no tempo discreto). O sinal de controle $u(k)$ que excitará os atuadores do sistema tem como função diminuir o erro $e(k)$ e manter o sistema estável.

$$
\begin{aligned}
u(k)= & {\left[K_{p} e(k)+\frac{K_{p}}{T_{i}}\left(\sum_{i=1}^{k} e(i)\right) T+\right.} \\
& \left.K_{p} T_{d}\left(\frac{e(k)-e(k-1)}{T}\right)\right]
\end{aligned}
$$

$K_{p}$ é o ganho proporcional, $T_{i}$ é o tempo integral, $T_{d}$ é o tempo derivativo e $T$ é o período de amostragem.

\subsection{O controlador PID com anti-windup}

Com o passar do tempo esta estrutura foi sendo aprimorada para resolver o problema do wind-up, que é observado quando se usa um controlador com ação integral. Quando o atuador chega a seu limite, a ação integral continua se acumulando e incrementando o sinal de controle, porém, sem provocar nenhuma mudança no atuador. Quando o erro mudar de sinal, 


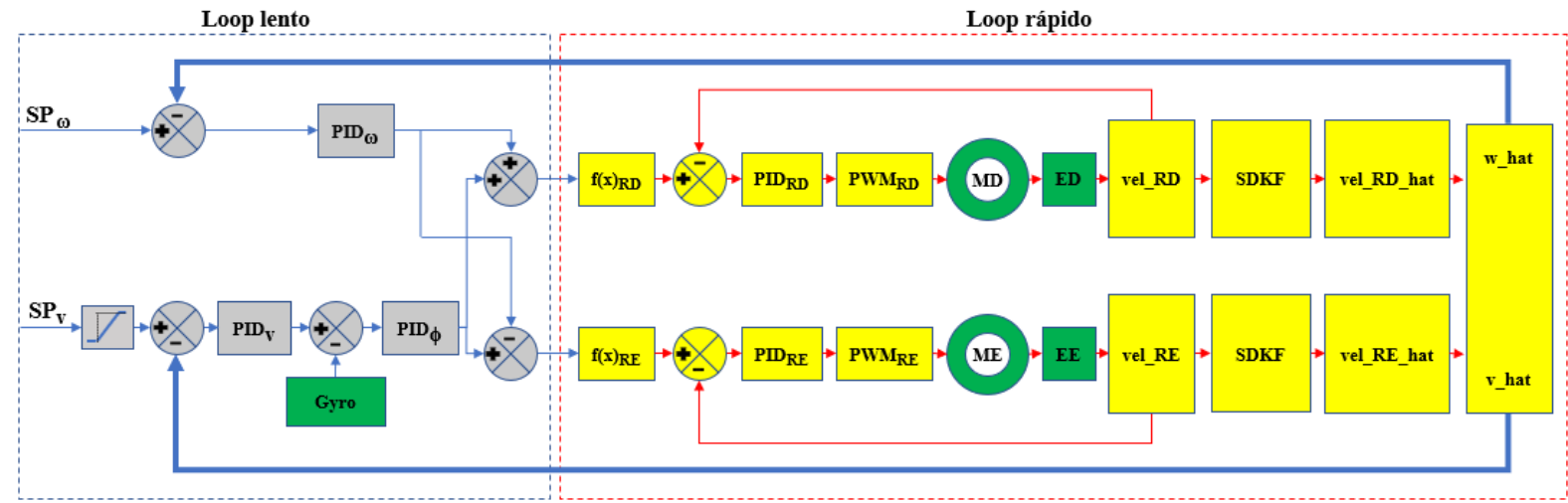

Figura 6 - Diagrama da estrutura do robô.

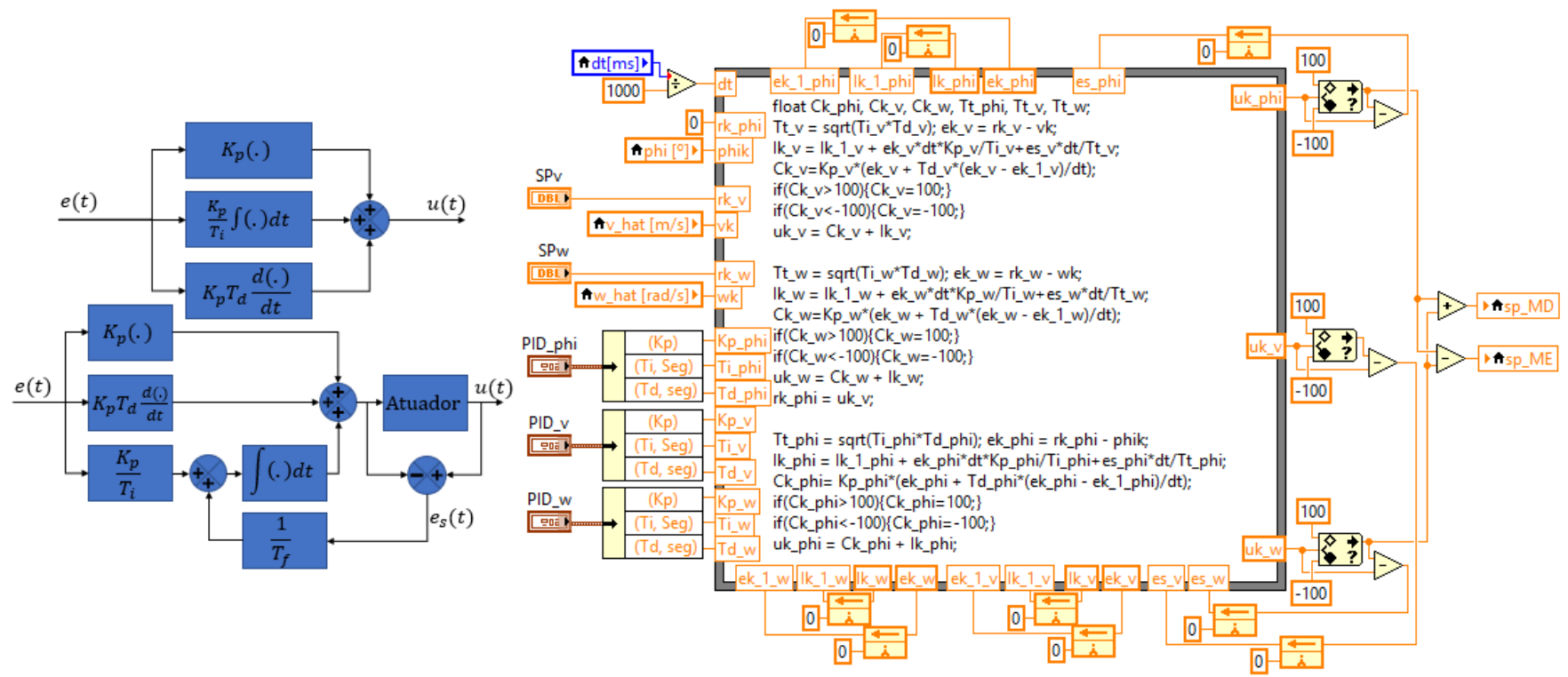

Figura 7 - Na esquerda, diagrama de blocos do controlador PID padrão ISA (acima) e com anti-windup (abaixo). Na direita, controladores de velocidades linear e angular, e de equilíbrio implementado no LabVIEW.

a ação integral provocará um atraso na mudança do sinal de controle, fazendo o sistema responder de forma lenta e oscilatória. Uma das técnicas para evitar este problema é a Back-Calculation and Tracking. Esta técnica implementa alterações na ação integral da estrutura padrão ISA (Figura 7), evitando que o sinal de controle se perca quando houver uma saturação do atuador, e isto pode ser conferido na equação (31) (também conhecido por PID com anti-windup).

$$
\begin{aligned}
u(k)= & {\left[K_{p} e(k)+K_{p} T_{d}\left(\frac{e(k)-e(k-1)}{T}\right)\right]+} \\
& \sum_{i=1}^{k}\left[\frac{K_{p}}{T_{i}} e(i)+\frac{1}{T_{f}} e_{s}(i)\right]
\end{aligned}
$$

\subsection{Estrutura genérica}

Pela Figura 6 pode-se observar o diagrama do robô contento os controladores, onde: $S P_{v}$ é o sinal de referência da velocidade linear, $S P_{\omega}$ é o sinal de referência da velocidade angular. O software foi desenvolvido seguindo este diagrama. O loop rápido é amostrado em $5 \mathrm{~ms}$ e o lento em $15 \mathrm{~ms}$.

Considerando a aplicação da abordagem clássica de controle automático, são necessários três controladores para realizar o controle de equilíbrio $\left(\mathrm{PID}_{\phi}\right)$, o controle da velocidade linear $\left(\mathrm{PID}_{\mathrm{v}}\right)$ e o controle da velocidade angular do robô (PID $\left.\omega\right)$.

Outros dois controladores são usados para fazer o controle de velocidade da roda do lado direto ( $\mathrm{PID}_{\mathrm{RD}}$ ) e do lado esquerdo (PID $\mathrm{PE}_{\mathrm{RE}}$, com o objetivo de fazer com que o motor direito (MD) e o motor esquerdo (ME) tenham respostas aproximadamente iguais para um mesmo sinal de referência, reduzindo os efeitos de atrito e agarramento das caixas de redução mecânica (gearbox). Os sinais de atuação desses controladores são convertidos em valores de duty cicle de PWM para os respectivos motores ( $P W M_{R D}$ e $\left.P W M_{R E}\right)$. Na sequência, os sinais dos encoders do lado direito (ED) e esquerdo (EE) são processados por meio da odometria e transformados em velocidade da roda direita (vel_RD) e velocidade da roda esquerda (vel_RE), que por sua vez, é filtrado pelo Kalman, como descrito na seção 3.2.

\subsubsection{Os controladores de equilíbrio e velocidade linear e angular}

Como pode ser visto na Figura 7, para resolver o problema de controle automático, foram implementados no LabVIEW os controladores responsáveis por regular o equilíbrio, a velocidade linear e a velocidade angular do robô, seguindo a abordagem do PID com anti-windup (equação (31)). Os parâmetros de sintonia foram obtidos por meio de tentativa e erro. Seus respectivos valores podem ser vistos na Figura 8 (PID_v, PID_w e PID_phi). 

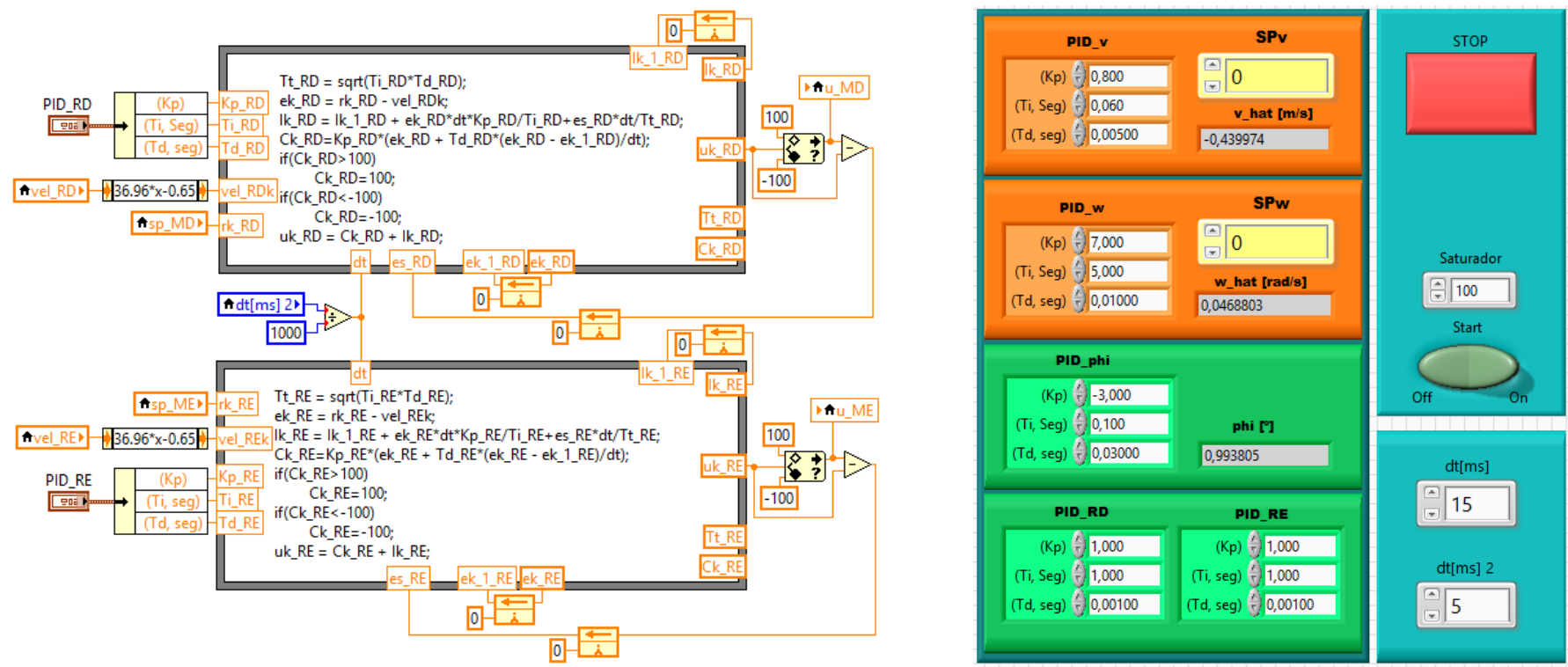

Figura 8 - Na esquerda, controladores de velocidade para cada roda. Na direita, foto do supervisório com os parâmetros de sintonia dos controladores implementados.

\subsubsection{Os controladores de velocidade da roda direita $e$ esquerda}

Pela Figura 6 pode-se observar a existência de duas malhas de controles internas (no lado do loop rápido). Cada malha resolve o problema de um de controle servo, onde as ações de controle são responsáveis por seguir a referência de velocidade da roda direita e da roda esquerda do robô. Por meio de testes, verificou-se que, para estes controladores, não haveria contribuição significativa em utilizar o sinal de velocidade filtrado pelo FK. Portanto, seguindo a abordagem do PID com anti-windup (equação (31)), como mostrado na Figura 8, os controladores foram implementados no LabVIEW. Os parâmetros de sintonia foram obtidos por meio de testes empíricos e seus respectivos valores podem também serem vistos na Figura 8 (PID_RD e PID_RE).

\section{RESULTADOS PRÁTICOS}

\subsection{Controladores Servo}

Nos testes práticos podem ser observados os comportamentos dos controladores servos (PID_RD e PID_RE). Na Figura 9 pode-se observar que, em malha fechada, quando a referência varia ao longo do tempo, a resposta obtida (linha tracejada) segue a referência, porém, com um erro que não interfere significativamente no controle do robô. Os valores negativos de duty cycle representam velocidades negativas das rodas.

\subsection{Controladores regulatórios}

Para os testes práticos da velocidade linear, foi implementada uma aproximação linear e contínua para a função de Heaviside, chamada de função rampa, com o objetivo de suavizar o degrau aplicado para a referência de velocidade linear $S P_{v}$.

Pode-se ver na Figura 10 que vários degraus foram aplicados ao longo de um período de teste, no entanto, o sistema apresentou uma resposta oscilatória para a velocidade
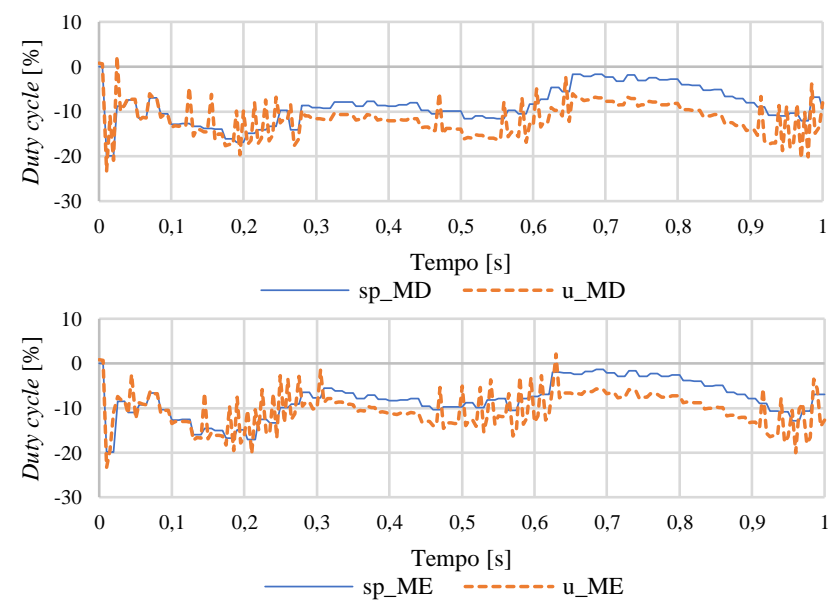

Figura 9 - Testes de resposta em malha fechada da velocidade da roda direita e roda esquerda quando é aplicado valores de duty cycle variados. A linha continua é a linha de referência, a linha tracejada é a resposta obtida.

linear, semelhante ao comportamento de um sistema de segunda ordem. A resposta ao degrau de velocidade angular se comportou como um sistema de primeira ordem ruidoso. Um vídeo dos testes pode ser visto em (GAIn 2019).

\section{CONCLUSÃO}

Neste trabalho foi apresentada a construção de um robô móvel de duas rodas auto equilibrado.

Como os encoders das rodas são de baixa resolução (230ppr), isso gera medições de velocidades ruidosas, logo, houve a necessidade de filtrar os ruídos para que seja feita a aplicação de controladores automáticos. O Filtro de Kalman de uma dimensão foi escolhido para esta tarefa, o SDKF, uma solução factível e de baixo custo computacional que se mostrou eficaz, como pode-se observar nas Figuras 3 e 5.

Após a aplicação do SDKF às medições de velocidades, seguese para a implementação dos controladores de acordo com a estrutura de diagrama de blocos apresentada na Figura 6. São 

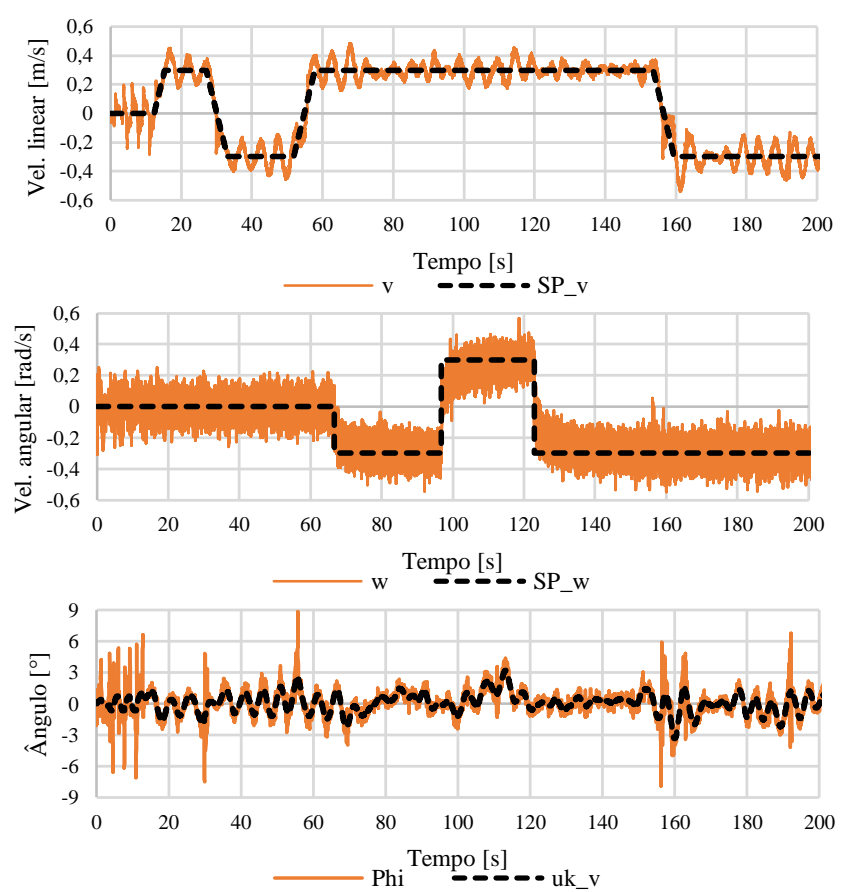

Figura 10 - Testes de resposta em malha fechada da velocidade linear e angular e equilíbrio do robô, quando é aplicado degraus variados. A linha tracejada é a referência, a linha continua é a resposta obtida.

aplicados cinco controladores do tipo PID com anti-windup: um para controle de velocidade do motor direito e esquerdo, um para o controle de equilíbrio do robô, um para o controle da velocidade de orientação e outro para a velocidade linear. A sintonia dos controladores foi feita com tentativa e erro.

Na Figura 9 pode ser observado o comportamento do controlador de cada roda, nela a resposta do sistema segue a o setpoint com um erro tolerável para a proposta deste trabalho. A Figura 10 apresenta os resultados dos testes realizados, onde pode-se observar que o equilíbrio se manteve o suficientemente estável frente a diferentes valores de velocidades linear e angular. Um vídeo do funcionamento pode ser visto em GAIn (2019).

Como recomendação para projetos futuros, pode-se aplicar lógica nebulosa (Fuzzy) ou o controlador Backstepping para o controle de trajetória, algoritmos para geração trajetória (Aestrela), algoritmo de reconhecimento de fala para responder a comandos de deslocamento, e outros.

\section{AGRADECIMENTOS}

Agradecemos ao CNPq, à SETEC/MEC, à FAPES, à Vale S.A., IFES e ao GAIn (Grupo de Automação Industrial) pelo apoio concedido às pesquisas que deram origem a este trabalho.

\section{REFERÊNCIAS BIBLIOGRÁFICAS}

Bishop, G. \& Welch, G. 2001, 'An Introduction to the Kalman Filter', Proc of SIGGRAPH, Course.

Campos, M.C.M.M. de \& Teixeira, H.C.G. 2006, Controles típicos de equipamentos e processos industriais, Editora Blucher (ed.), $1^{\mathrm{a}}$., São Paulo.

Dobra, A. 2015, 'General classification of robots. Size criteria', 23rd International Conference on Robotics in Alpe-Adria-Danube Region, IEEE RAAD 2014 Conference Proceedings.

GAIn, "Implementação do controle automático de velocidade linear e angular de um robô auto-equilibrado" 2019. [online]. Available: https://youtu.be/MyfWXMF0Uuk.

Grasser, F., D’Arrigo, A., Colombi, S. \& Rufer, A.C. 2002, 'JOE: A mobile, inverted pendulum', IEEE Transactions on Industrial Electronics, vol. 49, no. 1, pp. 107-14.

Ha, Y. \& Yuta, S. 1994, 'Trajectory tracking control for navigation of self-contained mobilelninverse pendulum', Proceedings of IEEE/RSJ International Conference on Intelligent Robots and Systems (IROS'94), vol. 3.

Kumar, S., Vijaydeep \& Gupta, P. 2017, 'Self-balancing vehicle using Kalman Filter', 2017 International Conference on Communication and Signal Processing (ICCSP), IEEE, pp. 1496-9.

National Instruments 2018, 'USER GUIDE AND SPECIFICATIONS', NI myRIO-1900, viewed <http://www.ni.com/pdf/manuals/376047c.pdf $>$.

del Pino, I., Á. Muñoz-Bañón, M., Á. Contreras, M., CovaRocamora, S., A. Candelas, F. \& Torres, F. 2018, 'Speed Estimation for Control of an Unmanned Ground Vehicle using Extremely Low Resolution Sensors', Proceedings of the 15th International Conference on Informatics in Control, Automation and Robotics, vol. 1, SCITEPRESS Science and Technology Publications, pp. 206-13.

Prasetio, B.H. 2016, 'Ensemble Kalman filter and PID controller implementation on self balancing robot', Proceedings - 2015 International Electronics Symposium: Emerging Technology in Electronic and Information, IES 2015, pp. 105-9.

Rosário, J.M. 2005, Princípios de mecatrônica, Pearson Educación.

Sadeghian, R. \& Masoule, M.T. 2016, 'An experimental study on the PID and Fuzzy-PID controllers on a designed twowheeled self-balancing autonomous robot', 2016 4th International Conference on Control, Instrumentation, and Automation, ICCIA 2016, pp. 313-8.

Sarathy, S., Hibah, M.M.M., Anusooya, S., Kalaivani, S. 2018, 'Implementation of Efficient Self Balancing Robot', IEEE International Conference on Recent Trends in Electrical, Control and Communication (RTECC), pp. 65-70.

Segway 2018, 'Segway Personal Transporter', User Manual, viewed 26 March 2018, <http://www.segway.com>.

Shaowei, W. \& Shanming, W. 2012, 'Velocity and acceleration computations by single-dimensional Kalman filter with adaptive noise variance', Przeglad Elektrotechniczny.

Shiroma, N., Matsumoto, O., Kajita, S. \& Tani, K. 1996, 'Cooperative behavior of a wheeled inverted pendulum for object transportation', Proceedings of IEEE/RSJ International Conference on Intelligent Robots and Systems. IROS '96, vol. 2, pp. 396-401. 